\title{
TI.125.1
}

\section{InCommon Steward Program FAQ}

- PDF: InCommonStewardProgramFAQ.pdf

- Text: InCommonStewardProgramFAQ.txt

\section{More Information}

\begin{tabular}{|l|l|}
\hline Repository ID & TI.125.1 \\
\hline Persistent URL & http://doi.org/10.26869/TI.125.1 \\
\hline Title & InCommon Steward Program FAQ \\
\hline Authors & Dean Woodbeck https://orcid.org/0000-0002-0996-1719 \\
\hline Sponsor & Internet2 Trust and Identity Executive Management \\
\hline Review & \\
\hline Status & Legacy \\
\hline Publish Date & 2016 \\
\hline DOI & $10.26869 / T I .125 .1$ \\
\hline Signature & \\
\hline Deprecated & No \\
\hline Future Review & \\
\hline Supersedes & \\
\hline Format & PDF, Text \\
\hline Related Docs & \\
\hline Development Location & \\
\hline IP Framework & This document was extracted from https://incommon.org/steward/faq.html. \\
\hline Subject Tags & \\
\hline Notes & \\
\hline
\end{tabular}

